\title{
Programmed self-assembly of DNA origami nanoblocks into anisotropic higher-order nanopatterns
}

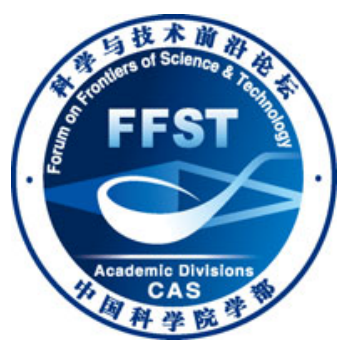

\author{
FU YanMing, CHAO Jie, LIU HuaJie* \& FAN ChunHai* \\ Laboratory of Physical Biology, Shanghai Institute of Applied Physics, Chinese Academy of Sciences, Shanghai 201800, China
}

Received July 16, 2012; accepted August 30, 2012; published online April 8, 2013

\begin{abstract}
Anisotropic nanopatterns have potentials in constructing novel plasmonic structures which have various applications in such as super-resolution microscopy, medicine, and sensors. However, it remains challenging to build big anisotropic nanopatterns that are suitable for big noble metal nanoparticles. Herein, we report a simple and reliable strategy for constructing DNA origami-based big anisotropic nanopatterns with controlled size and shape, nanoscale resolution, and fully addressability. Two kinds of basic DNA origami nanoblocks - cross-shaped and rectangular DNA origami units were used. We have demonstrated that by encoding nanoblocks' edges, anisotropic higher-order nanopatterns, such as dimer, trimer, tetramer and mini "windmill" like pentamer nanopatterns could be constructed. To show the potential use as template to direct the assembly of anisotropic nanoparticles arrays, a proof of concept work was conducted by anchoring streptavidin nanoparticles on the "windmill" template to form a chiral array. Significantly, these nanopatterns have the sizes of hundreds of nanometers, which are in principle also suitable for big noble metal nanoparticles arrays.
\end{abstract}

self-assembly, DNA origami, anisotropic nanopattern, nanoparticles array, plasmonic structure

Citation: Fu Y M, Chao J, Liu H J, et al. Programmed self-assembly of DNA origami nanoblocks into anisotropic higher-order nanopatterns. Chin Sci Bull, 2013, 58: 2646-2650, doi: 10.1007/s11434-012-5530-3

Recently, the emerging field of plasmonics has become one of the most interesting and active research areas in nanotechnology [1]. The amazing ability of dramatic alteration of incident light by the surface plasmon resonance property of noble metal nanostructures has enabled numerous applications in a variety of scientific disciplines [2-4], especially in such as super-resolution microscopy, medicine, and sensors [5,6]. Theoretical and experimental studies have demonstrated that the plasmonic property is highly dependent on the arrangement of noble metal nanoparticles [7]. Particularly, anisotropic nanoparticles arrays with increased complexity and hierarchy are expected to be promising in achieving novel optical properties. For example, anisotropic plasmonic structures can enhance the signal intensity of the Fano resonance [8]. However, it remains a great challenge for the construction of anisotropic structures in nanoscale precisely [9].

*Corresponding authors (email: liuhuajie@sinap.ac.cn; fchh@sinap.ac.cn)
Given the unparalleled molecular recognition and self-assembly ability, currently DNA has been proven as a powerful material in constructing elaborate nanostructures for controlled organizing natural or synthetic nanoparticles $[10,11]$. It is also believed that the DNA template-directed assembly could be an ideal approach for building plasmonic structures [12]. Especially, the new invented DNA origami technique has enabled the construction of fully addressable nanopatterns with sub-6 nm resolution [13,14]. An individual DNA origami structure has excellent coding ability that can be used as template for assembling small nanoparticles into arrays [15]. However, for building plasmonic structures, the size of origami monomer is still too small for big noble metal nanoparticles which could induce strong coupling.

Herein, we propose our strategy for constructing anisotropic higher-order DNA origami nanopatterns through programmed self-assembly of origami monomer nanoblocks with encoded edges. With the advantages of big size, fully addressable anisotropy, easy preparation, and monodisper- 
sity, these nanopatterns have the potential as templates for building anisotropic plasmonic structures.

\section{Materials and methods}

\subsection{Materials}

Streptavidin and all chemicals were bought from Sigma-Aldrich and used as received. All DNA sequences were synthesized and purified by Invitrogen China, Ltd. M13mp18 viral DNA was purchased from New England Biolabs, Inc.

\subsection{Preparation of DNA origami monomer nanoblocks}

Long scaffold M13mp18 DNA was mixed with synthesized short strands in $1 \times$ TAE-Mg buffer $(20 \mathrm{mmol} / \mathrm{L}$ Tris, $\mathrm{pH}$ 7.6, $2 \mathrm{mmol} / \mathrm{L}$ EDTA, $12.5 \mathrm{mmol} / \mathrm{L} \mathrm{MgCl}_{2}$ ). The final concentration is $32 \mathrm{nmol} / \mathrm{L}$ for each short strand, $3.2 \mathrm{nmol} / \mathrm{L}$ for M13mp18 DNA. The mixture was annealed from 60 to $35^{\circ} \mathrm{C}$ in $10 \mathrm{~min}$ on a PTC-200 Peltier Thermal Cycler (MJ Research).

\subsection{Preparation of higher-order DNA origami nanopatterns}

For preparation of anisotropic big nanopatterns, different DNA origami nanoblocks were mixed in equal volume and incubated at room temperature for $12 \mathrm{~h}$. For anchoring streptavidin nanoparticles, DNA origami-based anisotropic nanopatterns were mixed with excess streptavidin nanoparticles and incubated at $37^{\circ} \mathrm{C}$ for $10 \mathrm{~min}$.

For AFM measurements, a sample solution $(5 \mu \mathrm{L})$ was deposited onto a freshly cleaved mica surface and left to adsorb for $3 \mathrm{~min}$. $1 \times$ TAE-Mg buffer $(30 \mu \mathrm{L})$ was added to a liquid cell and the sample was scanned in a tapping mode using a $\mathrm{J}$ scanner of a Multi-mode Nanoscope IIIa AFM (Vecco/Digital Instruments) with a silicon nitride cantilever with sharpened pyramidal tip (OMCL-TR400PSA, Olympus).

\section{Results and discussion}

\subsection{Design principle}

In principle, a DNA origami monomer is formed through the guided folding of a long scaffold DNA, i.e. M13mp18 viral DNA in our case, with hundreds of short synthesized oligonucleotides into a specific nanopattern. Since the sequence is unique on whole origami, an origami monomer is therefore a fully anisotropic nanostructure. However, the size of each origami monomer is dependent on the length of the long scaffold. In the case of M13mp18, the size of an origami monomer (origami designs with holes [16] are ex- cluded) is $\sim 7 \times 10^{4} \mathrm{~nm}^{2}$, which is too small for directing the assembly of big noble metal nanoparticles, e.g. $50 \mathrm{~nm} \mathrm{Au}$ nanoparticles, into plasmonic structures. As an effort to overcome this disadvantage, strategies for assembling origami monomers into higher-order big nanopatterns have been reported. However, with few exceptions [17], the reported works were all concentrated on periodical origami arrays and these big nanopatterns on the whole have no anisotropy [18-20].

Like DNA origami monomers, the key characteristics of anisotropic higher-order DNA origami nanopatterns could be considered as that each position on the big nanopattern can be independently addressed and coded, and no repeated coding regions can be found on the whole pattern. A simple strategy to reach this goal is to assemble differently folded DNA origami units into a big nanopattern. But disadvantages of this strategy are also obvious, such as high price, increased difficulties in origami preparation, and increased complexity in higher-order assembly. An alternative strategy is to use DNA origami nanoblocks with same cores but differently encoded edges for anisotropic assembly. As shown in Figures 1, S1-S7 and S13-S15, only two kinds of basic DNA origami nanoblocks are used in this work-a cross-shaped four-way nanoblock [20] and a rectangular two-way nanoblock [21].

Different from DNA tile-based self-assembly structures [22], there are more strands suitable for encoding on each edge of a DNA origami nanoblock, which allow more freedom in design. For example, a basic rectangular DNA origami could generate a library of $2^{48}$ fully addressable derived nanoblocks. All of them share the same core from the original rectangular DNA origami but have various encoded edges. By connecting the corresponding encoded edges,

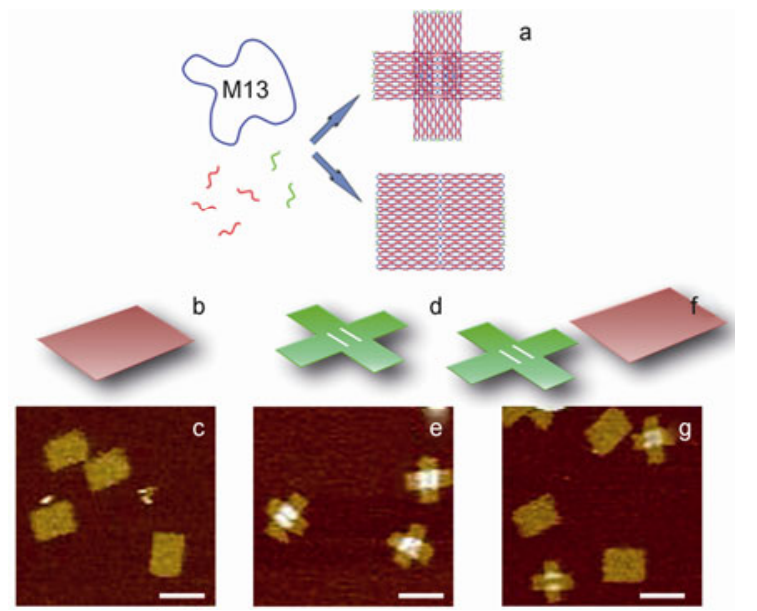

Figure 1 DNA origami nanoblocks and their AFM images. a, Schematic illustration of nanoblocks preparation; b and c, rectangular nanoblocks; d and e, cross-shaped nanoblocks with clearly visible seams; f and g, mixture of two nanoblocks. For the preparation of these nanoblocks, long scaffold M13mp18 DNA was mixed with synthesized short strands in $1 \times$ TAE-Mg buffer. The final concentration is $32 \mathrm{nmol} / \mathrm{L}$ for each short strand, 3.2 $\mathrm{nmol} / \mathrm{L}$ for M13mp18 DNA. The mixture was annealed from 60 to $35^{\circ} \mathrm{C}$ in $10 \mathrm{~min}$. Scale bars: $100 \mathrm{~nm}$. 
anisotropic higher-order self-assembly of DNA origami could be achieved.

\subsection{Anisotropic nanopatterns using cross-shaped origami nanoblocks}

Firstly, we tried to use cross-shaped origami nanoblocks to construct a simple dimer nanopattern. In order to encode edges, we designed a lot of parallel $5 \mathrm{nt}$ overhangs, which we call linkers and are stretched from origami nanoblock's internal parallel rows, on each edge. Each linker sequence is unique and therefore by designed combination, the edge could be encoded for a specific connection to its corresponding encoded edge. In the case of dimer nanopattern, each cross-shaped nanoblock has one encoded edge, which is correlated to the encoded edge of the other nanoblock. As shown in Figure 2a and $\mathrm{b}$ and Figures S8 and S16, dimer nanopatterns with length of $200 \mathrm{~nm}$ and width of $100 \mathrm{~nm}$ could be found by AFM measurements. Especially, the seams of these two cross are perpendicular to each other, in accord with the design, proving that this strategy can achieve anisotropic higher-order self-assembly.

Further we increased the number of nanoblocks for constructing a more complex 2D tetramer nanopattern. As shown in Figures 2c and S9, four cross-shaped nanoblocks were employed and each nanoblock has two encoded edges. Figures 2d and S17 show an AFM image of the tetramer nanopattern, which has the same length and width of 200 $\mathrm{nm}$. Although the appearance of this tetramer seems to have $C_{4}$ symmetry, actually it is addressable at any position and highly anisotropic.

\subsection{Anisotropic nanopatterns using cross-shaped and rectangular origami nanoblocks}

Next, rectangular DNA origami nanoblocks were employed in order to increase the diversity and complexity of the higher-order self-assembly. In the simplest case, a rectangular nanoblock was connected to a cross-shaped nanoblock

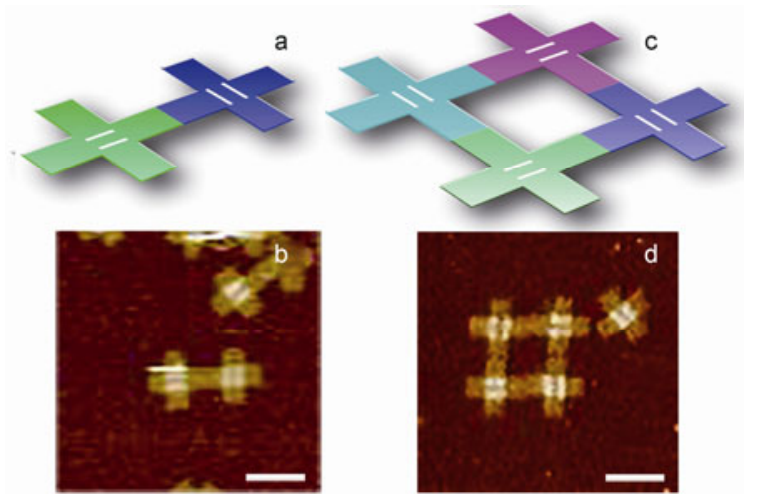

Figure 2 Anisotropic self-assembly using cross-shaped DNA origami nanoblocks. Different DNA origami nanoblocks were mixed in equal volume and incubated at room temperature for $12 \mathrm{~h}$. Scale bars: $100 \mathrm{~nm}$. to form a dimer (Figures 3a and S10). It is worth noting that the number of linkers on the edge of rectangular nanoblock is more than that on the cross-shaped nanoblock's edge. Therefore, in the present design, we only used the middle part of the linkers on the rectangular nanoblock's edge. However, it is also possible to use linkers on the top or bottom part to enable more dimer nanopatterns formation. From the AFM image (Figures $3 b$ and S18), it is clear that this dimer has a length of $200 \mathrm{~nm}$ and a width of $100 \mathrm{~nm}$, and the seam of the cross-shaped nanoblock is parallel to the short edge of the rectangular nanoblock.

Similarly, a more complex trimer nanopattern could be constructed by the self-assembly of two cross-shaped nanoblocks with one rectangular nanoblock (Figures $3 \mathrm{c}$ and $\mathrm{S} 11$ ). Figures $3 \mathrm{~d}$ and S19 show the AFM image of this trimer, which has a rectangular core with two cross-shaped arms. The length of this trimer is $300 \mathrm{~nm}$ and the width is still $100 \mathrm{~nm}$

\subsection{A DNA origami "windmill" for anisotropic patterning of nanoparticles into a chiral array}

Furthermore, a cross-shaped nanoblock was used as core to enable the connection of four rectangular nanoblocks on its four arms to build a "windmill" nanopattern. As shown in Figures 4a and S12, each edge of the cross-shaped nanoblock is connected to the middle part of a rectangular nanoblock's edge. From the AFM results (Figures $4 \mathrm{~b}$ and S20), this pentamer nanopattern can be successfully constructed, which looks like a mini "windmill" with both length and width of $300 \mathrm{~nm}$. Like the tetramer nanopattern shown in Figures 2d, although this pentamer is highly symmetrical in appearance, it is indeed an anisotropic nanopattern with the inherent addressability for anchoring nanoparticles with nanoscale resolution and at the single-particle level.

In order to show its intrinsic anisotropy and the potential as template to direct the assembly of nanoparticles into

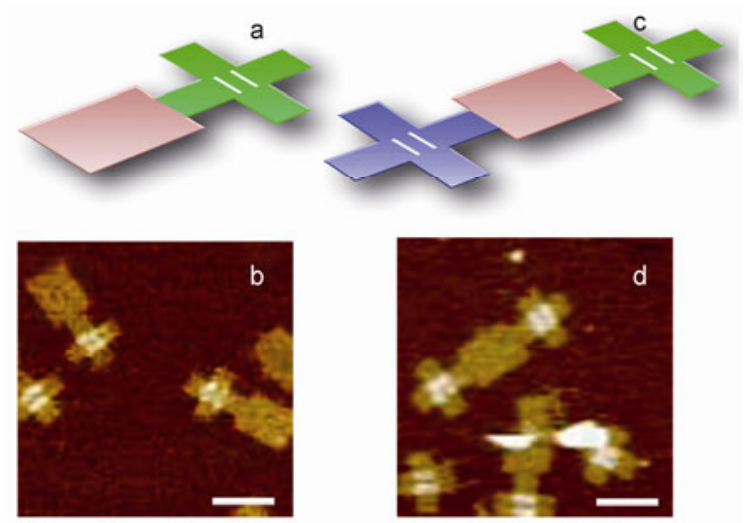

Figure 3 Anisotropic self-assembly using cross and rectangular DNA origami nanoblocks. Scale bars: $100 \mathrm{~nm}$. Different DNA origami nanoblocks were mixed in equal volume and incubated at room temperature for $12 \mathrm{~h}$. Scale bars: $100 \mathrm{~nm}$. 


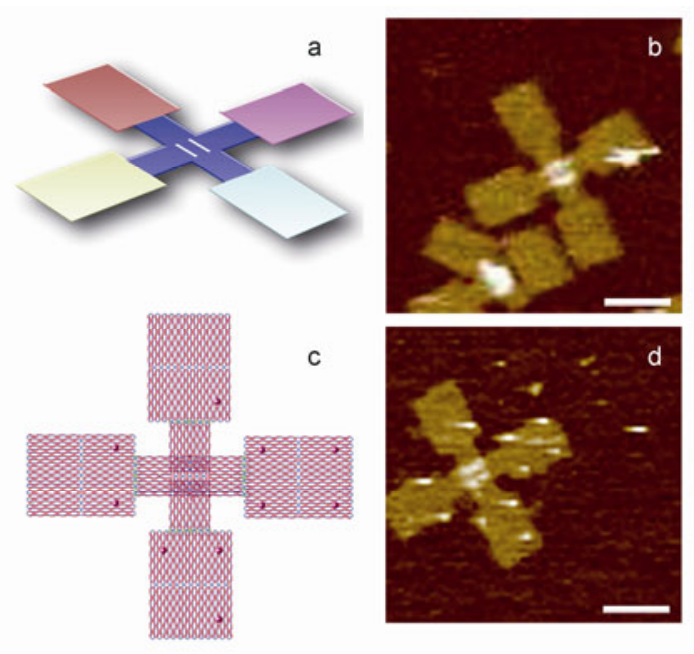

Figure 4 A DNA origami "windmill". Scale bars: $100 \mathrm{~nm}$. Different DNA origami nanoblocks were mixed in equal volume and incubated at room temperature for $12 \mathrm{~h}$. For anchoring streptavidin nanoparticles, DNA origami-based anisotropic nanopatterns were mixed with excess streptavidin nanoparticles and incubated at $37^{\circ} \mathrm{C}$ for $10 \mathrm{~min}$. Scale bars: $100 \mathrm{~nm}$.

plasmonic structures, we conducted a proof of concept work by building a chiral nanoparticles array on this template (Figure 4c). For experimental convenience, streptavidin nanoparticles were used. From the AFM image (Figure 4d) we can see that the streptavidin modification on the "windmill" is exactly same as the design, proving that this nanopattern is fully addressable and inherently anisotropic.

\section{Conclusions}

In summary, based on rectangular and cross-shaped DNA origami nanoblocks, using programmable self-assembly we successfully constructed anisotropic nanopatterns with different size and complexity. We proved that these nanopatterns could be templates for anchoring nanoparticles into anisotropic arrays. This programmable self-assembly construction method is simple and reliable. The size and shape of the assembled nanopattern could be varied by changing the number and shape of basic nanoblocks. When using as template for nanoparticles, the inter-particle distance and arrangement could be easily adjusted. Most importantly, these big nanopatterns are suitable for big nanoparticles with diameters up to $100 \mathrm{~nm}$, which is a key point for building practical plasmonic nanostructures. In addition, it is also expected that this method could be useful for other applications, such as medical diagnosis, sensors and photonic circuits.
This work was supported by the National Basic Research Program of China (2012CB932600), the National Natural Science Foundation of China (20725516, 90913014, 21028005 and 21103219), Shanghai Pujiang Program (11PJ1412000) and the Chinese Academy of Sciences.

1 Odom T W, Schatz G C. Introduction to plasmonics. Chem Rev, 2011, 111: 3667-3668

2 Jones M R, Osberg K D, Macfarlane R J, et al. Templated techniques for the synthesis and assembly of plasmonic nanostructures. Chem Rev, 2011, 111: 3736-3827

3 Wang S, Jin J, Gao W, et al. Study on surface-enhanced fluorescence of gold nanorods coated with polyelectrolyte multilayers. Chin Sci Bull, 2012, 57: 408-411

$4 \mathrm{Li} \mathrm{Z,} \mathrm{Li} \mathrm{J.} \mathrm{Recent} \mathrm{progress} \mathrm{in} \mathrm{engineering} \mathrm{and} \mathrm{application} \mathrm{of} \mathrm{surface}$ plasmon resonance in metal nanostructures. Chin Sci Bull, 2011, 56: 2631-2661

5 Cortie M B, McDonagh A M. Synthesis and optical properties of hybrid and alloy plasmonic nanoparticles. Chem Rev, 2011, 111: 37133735

6 Anker J N, Hall W P, Lyandres O, et al. Biosensing with plasmonic nanosensors. Nat Mater, 2008, 7: 442-453

7 Ghosh S K, Pal T. Interparticle coupling effect on the surface plasmon resonance of gold nanoparticles: From theory to applications. Chem Rev, 2007, 107: 4797-4862

8 Luk'yanchuk B, Zheludev N I, Maier S A, et al. The Fano resonance in plasmonic nanostructures and metamaterials. Nat Mater, 2010, 9: 707-715

9 Akcora P, Liu H, Kumar S K, et al. Anisotropic self-assembly of spherical polymer-grafted nanoparticles. Nat Mater, 2009, 8: 354-359

10 Aldaye F A, Palmer A L, Sleiman H F. Assembling materials with DNA as the guide. Science, 2008, 321: 1795-1799

11 Zhang $\mathrm{C}$, Yang J, Xu J. Molecular logic computing model based on self-assembly of DNA nanoparticles. Chin Sci Bull, 2011, 56: 35663571

12 Tan S J, Campolongo M J, Luo D, et al. Building plasmonic nanostructures with DNA. Nat Nanotechnol, 2011, 6: 268-276

13 Rothemund P W. Folding DNA to create nanoscale shapes and patterns. Nature, 2006, 440: 297-302

14 Qian L, Wang Y, Zhang Z, et al. Analogic China map constructed by DNA. Chin Sci Bull, 2006, 51: 2973-2976

15 Shen X, Song C, Wang J, et al. Rolling up gold nanoparticle-dressed DNA origami into three-dimensional plasmonic chiral nanostructures. J Am Chem Soc, 2012, 134: 146-149

16 Fu Y, Pei H, Zeng D, et al. An scaffolded DNA origami algorithm for polygon network structures and hollow three-dimensional structures. Acta Biophys Sin, 2010, 26: 705-755

17 Endo M, Sugita T, Rajendran A, et al. Two-dimensional DNA origami assemblies using a four-way connector. Chem Commun, 2011, 47: 3213-3215

18 Li Z, Liu M, Wang L, et al. Molecular behavior of DNA origami in higher-order self-assembly. J Am Chem Soc, 2010, 132: 1354513552

19 Zhao Z, Yan H, Liu Y. A route to scale up DNA origami using DNA tiles as folding staples. Angew Chem Int Ed, 2010, 49: 1414-1417

20 Liu W, Zhong H, Wang R, et al. Crystalline two-dimensional DNA-origami arrays. Angew Chem Int Ed, 2011, 50: 264-267

21 Ke Y, Lindsay S, Chang Y, et al. Self-assembled water-soluble nucleic acid probe tiles for label-free RNA hybridization assays. Science, 2008, 319: 180-183

22 Liu Y, Ke Y, Yan H. Self-assembly of symmetric finite-size DNA nanoarrays. J Am Chem Soc, 2005, 127: 17140-17141

Open Access This article is distributed under the terms of the Creative Commons Attribution License which permits any use, distribution, and reproduction in any medium, provided the original author(s) and source are credited. 


\section{Supporting Information}

\section{Schematic illustrations}

Figure S1 Cross-shaped origami nanoblock A-(D-R-L-U).

Figure S2 Cross-shaped origami nanoblock B-(D-R-L-U).

Figure S3 Rectangular origami nanoblock A-R.

Figure S4 Rectangular origami nanoblock B-D.

Figure S5 Rectangular origami nanoblock B-L.

Figure S6 Rectangular origami nanoblock B-R.

Figure S7 Rectangular origami nanoblock B-U.

Figure S8 Dimer nanopattern assembled from two cross-shaped nanoblocks.

Figure S9 Tetramer nanopattern assembled from four cross-shaped nanoblocks.

Figure S10 Dimer nanopattern assembled from one cross-shaped nanoblock and one rectangular nanoblock.

Figure S11 Trimer nanopattern assembled from two cross-shaped nanoblocks and one rectangular nanoblock.

Figure S12 Pentamer "windmill" nanopattern assembled from one cross-shaped nanoblock and four rectangular nanoblocks.

Figure S13 Cross-shaped DNA origami nanoblocks.

Figure S14 Rectangular DNA origami nanoblocks.

Figure S15 The simple mixture of rectangular and cross-shaped DNA origami nanoblocks.

Figure S16 Dimer nanopattern assembled from two cross-shaped nanoblocks.

Figure S17 Tetramer nanopattern assembled from four cross-shaped nanoblocks.

Figure S18 Dimer nanopattern assembled from one cross-shaped nanoblock and one rectangular nanoblock.

Figure S19 Trimer nanopattern assembled from two cross-shaped nanoblocks and one rectangular nanoblock.

Figure S20 Pentamer “windmill” nanopattern.

\section{Additional AFM images (scale bars: $100 \mathrm{~nm}$ )}

\section{DNA sequences}

The supporting information is available online at csb.scichina.com and www.springerlink.com. The supporting materials are published as submitted, without typesetting or editing. The responsibility for scientific accuracy and content remains entirely with the authors. 